\title{
Low ALT Levels Independently Associated with 22-Year All-Cause Mortality Among Coronary Heart Disease Patients
}

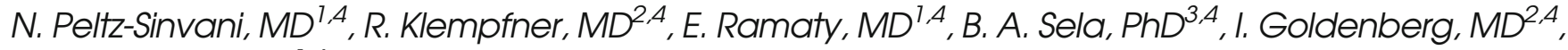 \\ and $G$. Segal, $M D^{1,4}$
}

'Department of Internal Medicine "T", Chaim Sheba Medical Center, Ramat Gan, Israel; 'Leviev Heart Institute, Chaim Sheba Medical Center, Ramat Gan, Israel; ${ }^{3}$ Institute of Chemical Pathology, Chaim Sheba Medical Center, Ramat Gan, Israel; ${ }^{4}$ Sackler Faculty of Medicine, Tel Aviv University, Tel Aviv, Israel.

BACKGROUND: Low alanine aminotransferase (ALT) blood levels are known to be associated with frailty and increased risk of long-term mortality in certain populations. However, the contribution of this marker to longterm outcome has not been assessed in patients with chronic coronary heart disease.

OBJECTIVE: The aim of the current study was to assess the association between low ALT values and long-term, 22.8-year, all-cause mortality in this population.

PARTICIPANTS: We examined the association of low ALT $(<17$ IU/1) with long-term all-cause mortality in the Bezafibrate Infarction Prevention (BIP) Registry population.

KEY RESULTS: Appropriate laboratory and survival data were available for 6,575 patients, without known liver pathology, included in the BIP registry, with a median follow-up period of 22.8 years. The cumulative probability of all-cause mortality was significantly higher in the low ALT group compared with patients with higher ALT levels (65.6\% vs. $58.4 \%$; log-rank $p<0.001$ ). Consistently, multivariate analysis, adjusted for multiple established predictors of mortality in this population, demonstrated that low ALT is independently associated with $11 \%$ greater long-term (22.8 years) mortality risk [HR 1.11 (95\% confidence interval: 1.03-1.19; adjusted $p<0.01)]$.

CONCLUSIONS: Low ALT levels are associated with increased long-term mortality among middle-aged patients with stable coronary heart disease. This association remained statistically significant after adjustment for other well-established risk factors for mortality in this population.

KEY WORDS: aging; cardiovascular disease; life course; risk assessment; screening; survey research.

$\mathrm{J}$ Gen Intern Med 31(2):209-14

DOI: $10.1007 / \mathrm{s} 11606-015-3480-6$

(C) Society of General Internal Medicine 2015

The authors N. Peltz-Sinvani and R. Klempfner contributed equally to the study

Received March 9, 2015

Revised June 1, 2015

Accepted July 13, 2015

Published online August 6, 2015

\section{INTRODUCTION}

Several essential metabolic steps in the liver and skeletal muscles are facilitated by the enzyme alanine aminotransferase (ALT), which catalyzes the transformation of keto acids such as pyruvate into amino acids such as alanine. In contrast to the known etiology and consequences of increased blood levels of ALT [as well as aspartate aminotransferase (AST)], as in hepatitis, less is known with regard to cases of lower than normal ALT values in the blood. Low ALT levels were found to be a biomarker for increased frailty and subsequent increased risk of mortality in old age $\mathrm{e}^{1,2}$ and among chronic kidney disease $[\mathrm{CKD}]$ patients treated with hemodialysis. ${ }^{3,4}$ Frailty, as a qualitative definition, currently diagnosed by several indices, ${ }^{5}$ is thought to be associated with decreased skeletal muscle mass. ${ }^{1,6,7}$ Therefore, it is plausible to assume that low ALT levels are associated with sarcopenia. ${ }^{1,7}$ Nevertheless, a causative relation between low ALT and shortened life span has not yet been established.

The authors of this publication have recently demonstrated that low-normal ALT values $(<17 \mathrm{IU} / \mathrm{l})$ are associated with increased risk of long-term mortality in large, selected populations. Among others, this association was statistically significant among middle-aged adults attending an annual preventive medicine clinic. ${ }^{8}$ Furthermore, in our study population, ALT $<17$ represented the lower quartile.

The Bezafibrate Infarction Prevention (BIP) study, a multicenter, double-blind, placebo-controlled randomized clinical trial, was conducted between February 1990 and October 1992 and included 15,524 patients aged 40 to 74 with stable coronary heart disease (CHD). The design and rationale of the BIP study have been published previously. ${ }^{9,10}$ The BIP registry patients had a diagnosis of CHD established by: (1) a history of myocardial infarction (MI) occurring no less than 6 months but no longer than 5 years prior to enrollment; (2) stable angina pectoris, which was symptomatic and documented on nuclear study/exercise test/coronary angiography (demonstrating a stenosis of above or equal to $60 \%$ in one of the major coronary arteries); (3) primary transcutaneous coronary angioplasty (PTCA) or coronary artery bypass graft surgery $(\mathrm{CABG})$ operation in the 6 months prior to enrollment.

We undertook the task of establishing a quantitative measure for frailty evaluation among stable CHD patients. The 
BIP registry patients, followed for over 2 decades, today represent a large cohort that is continuously aging. In the face of the advanced and novel techniques currently available for the treatment of coronary atherosclerosis and consequent heart failure, it seems valuable to better assess the frailty status of stable CHD patients. ${ }^{11,12}$ We took the opportunity to reaffirm our assumptions regarding the value of low ALT levels as a prognostic marker in this important patient population.

\section{METHODS}

\section{Study Population}

A total of 15,524 patients underwent screening for participation in the Bezafibrate Infarction Prevention (BIP) trial. The BIP patient population was aged 40 to 74 years with a diagnosis of CHD based on the following parameters: (1) documented myocardial infarction in the previous 5 years; (2) stable angina pectoris and a positive exercise test, positive myocardial ischemia by scintigraphy or $\geq 60 \%$ stenosis of one of the major coronary arteries (demonstrated by coronary angiography); or (3) documented PTCA or CABG operation in the 6 months prior to study enrollment. Within this database, patients with established liver disease, patients whose ALT levels exceeded $40 \mathrm{IU}$ (the upper normal limit, over which increased ALT levels are indicative of hepatocellular damage) and patients for whom no baseline ALT levels were available were excluded from the study. The remaining 6,575 cases with documented ALT levels of $40 \mathrm{IU}$ or less comprised the present study population.

\section{Clinical Information and Laboratory Assessment}

During the first visit, records were obtained concerning previous and current illnesses and medications used by the patient, and a complete physical examination was performed. Blood samples were taken after $\geq 12 \mathrm{~h}$ of fasting. ALT levels were tested using the standard laboratory measurements used during the BIP recruitment period. It is noted that the measurement technique changed during the following years; nevertheless, reference values for normal ALT activity were not changed.

\section{Outcomes and Definitions}

The primary outcome was all-cause mortality at a median follow-up duration of 22.8 years. Mortality data were obtained from the national Israeli population registry.

\section{Statistical Analysis}

The study population was divided into two prespecified groups based on the ALT levels obtained during the baseline visit with a cutoff value of $17 \mathrm{IU} / \mathrm{l}$. We compared the low ALT group (ALT $<17 \mathrm{IU} / 1$ ) to the ALT $\geq 17 \mathrm{IU} / 1$ group. Comparison of categorical variables was performed with chi-square analysis, and comparison of continuous variables was performed with the Student's t-test for variables with normal distribution and by the Kruskal-Wallis test for those that violated the normality assumption. The cumulative probability survival estimates for the 22.8-year outcome according to ALT category were constructed according to the Kaplan-Meier method and compared using the log-rank test. An additional analysis as described above was performed for deciles of ALT.

Logistic regression analysis, comparing the two ALT groups, was performed in order to identify independent predictors associated with low ALT values. The multivariate model included the following prespecified covariates: age, gender, body mass index (BMI), height, past MI, New York Heart Association (NYHA) functional status and baseline serum creatinine values.

The independent association of low ALT values and the allcause mortality outcome was explored using multivariate Cox proportional hazard regression analysis. The set of covariates included in the multivariate model was identified using a best subsets analysis. The covariates introduced were the following: age, gender, BMI, LDL cholesterol, glucose, triglycerides, creatinine concentration, alkaline phosphatase, hypertension, NYHA functional class, chronic obstructive pulmonary disease (COPD), prior myocardial infarction and smoking status. Additional analysis included the addition of AST and CPK as continuous variables using the above-described model.

All $p$ values were two sided, and a $p$ value lower than 0.05 was considered significant. The statistical software used was SPSS version 20 (IBM Inc.).

\section{RESULTS}

The baseline characteristics of 6,575 adult males and females are presented according to the ALT blood level grouping (Table 1). The mean age for the low ALT group was 61.31 years, while in the higher ALT group (18-40 IU/l) it was 59.24 years $(p<0.001)$. Female gender was more prevalent in the low ALT group ( $16.8 \%$ vs. $10.0 \% ; p<0.001)$. BMI values were lower in the low ALT group (25.8 vs. 26.6; $p=$ 0.01 ). The CPK blood concentration was significantly lower in the low ALT group (80.4 IU/1 vs. $103.4 \mathrm{IU} / 1 ; p<0.001)$.

\section{Predictors of Low ALT}

Independent predictors of low ALT values (Table 2) were older age, lower BMI, female gender and lower height. Creatinine concentration, NYHA functional class greater than 1 and past MI were not found to be independent predictors for low ALT values (Table 3).

\section{Long-Term Outcomes}

Among patients who had low ALT values at baseline, the overall unadjusted cumulative mortality probability was significantly higher than for patients who had higher ALT values 
Table 1 Study Population Baseline Characteristics Stratified by Normal and Lower-Than-Normal ALT Values

\begin{tabular}{|c|c|c|c|}
\hline \multirow[t]{2}{*}{ Characteristic } & \multicolumn{2}{|l|}{ ALT groups } & \multirow[t]{2}{*}{$p$ value } \\
\hline & $0<$ ALT $<17$ IU/I $(\mathrm{N}=1,889)$ & $17 \leq \mathrm{ALT} \leq 40 \mathrm{IU} / \mathrm{l}(\mathrm{N}=4,686)$ & \\
\hline Age [years, median (IQR)] & $61.31(54.81-67.81)$ & $59.24(52.22-66.25)$ & $<0.001$ \\
\hline Male gender [number (\%)] & $1571(83.2)$ & $4,216(90)$ & $<0.001$ \\
\hline BMI [median (IQR)] & $25.8(23.9-27.8)$ & $26.6(24.7-28.9)$ & 0.01 \\
\hline Arterial HTN [number (\%)] & $658(35)$ & $1458(31)$ & 0.003 \\
\hline Diabetes mellitus [number (\%)] & $278(15)$ & $760(16)$ & 0.13 \\
\hline Current smoker [number (\%)] & 248 (13) & $497(11)$ & 0.003 \\
\hline Past smoker [number (\%)] & $958(51)$ & $2723(58)$ & $<0.001$ \\
\hline Past AMI [number (\%)] & $1452(77)$ & $3498(75)$ & 0.39 \\
\hline Past CVA [number (\%)] & $27(1.4)$ & $55(1.2)$ & 0.41 \\
\hline COPD [number $(\%)]$ & $69(3.7)$ & $121(2.6)$ & 0.02 \\
\hline PVD [number (\%)] & $68(3.6)$ & $153(3.3)$ & 0.49 \\
\hline NYHA I [number (\%)] & $1342(73)$ & 3409 (74) & 0.71 \\
\hline NYHA II [number $(\%)]$ & $409(22)$ & $964(21)$ & 0.70 \\
\hline NYHA III-IV [number (\%)] & $90(4.9)$ & $215(4.7)$ & 0.74 \\
\hline Creatinine $(\mathrm{mg} / \mathrm{dl})$ & $1.16 \pm 0.21$ & $1.15 \pm 0.22$ & 0.11 \\
\hline ALT (IU/l) & $13.97 \pm 2.36$ & $24.33 \pm 5.60$ & $<0.001$ \\
\hline AST (IU/l) & $17.98 \pm 4.19$ & $22.40 \pm 4.73$ & $<0.001$ \\
\hline Glucose $(\mathrm{mg} / \mathrm{dl})$ & $106 \pm 35$ & $110 \pm 36$ & $<0.001$ \\
\hline CPK (IU/1) & $80.43 \pm 53$ & $103.4 \pm 68.7$ & $<0.001$ \\
\hline LDL [mg/dl, median (IQR)] & $155(139-173)$ & $155(139-172)$ & 0.61 \\
\hline HDL [mg/dl, median (IQR) $]$ & $34.8(31-39.5)$ & $34.5(30-39)$ & 0.15 \\
\hline TG [mg/dl, median (IQR)] & $136(105-175)$ & $142(110-185)$ & $<0.001$ \\
\hline Alkaline phosphatase (IU/1) & $187 \pm 63$ & $183 \pm 54$ & 0.42 \\
\hline
\end{tabular}

Data presented as numbers (\%) and median (IQR) or mean \pm SD as appropriate. BMI, body mass index; HTN, hypertension; AMI, acute myocardial infarction; CVA, cerebral vascular accident; COPD, chronic obstructive pulmonary disease; PVD, peripheral vascular disease; NYHA, New York Heart Association; ALT, alanine aminotransferase; AST, aspartate aminotransferase; CPK, creatinine phosphokinase; LDL, low density lipoprotein; HDL, high density lipoprotein; TG, triglycerides

at baseline (65.6 \% vs. $58.4 \%$, log-rank $p<0.001$; Fig. 1$)$. A graded mortality risk was observed within the normal ALT range, with mortality probability ranging from $69 \%$ in the lowest ALT decile to a $56 \%$ lower risk in the highest ALT decile, an absolute $13 \%$ difference (Fig. 2; $p$ value for trend $<0.001$ ).

In a multivariate analysis, the independent adjusted risk of mortality was higher for those who had ALT levels lower than 17 IU/1 (HR 1.11; $95 \%$ CI, 1.03-1.19, $p=0.01)$. Additional factors associated with independent long-term mortality were older age (per 1-year increment HR 1.08; 95 \% CI, 1.07-1.09, $p<0.001$ ), greater BMI (per unit increment HR 1.02; $95 \% \mathrm{CI}$, 1.01-1.03), smoked at enrollment (HR 1.71, $95 \%$ CI 1.541.91), diagnosis of hypertension (HR 1.09; $95 \%$ CI 1.02 1.17), past MI (HR 1.43;95 \% CI, 1.32-1.54), NYHA class $>1$ (HR 1.14, $95 \%$ CI, 1.08-1.20) and increased creatinine blood concentration (per $1 \mathrm{mg} / \mathrm{dl}$ increment HR 1.61, $95 \%$ CI, 1.38 1.87). When AST and CPK values were introduced into the

Table 2 Independent Predictors of Low Baseline ALT Values (<17 IU/I). **Logistic Regression Model Further Adjusted for Smoking Status and Serum Glucose Level

\begin{tabular}{lcccc}
\hline \hline & $\begin{array}{c}\text { Adjusted } \\
\text { odds ratio }\end{array}$ & \multicolumn{2}{c}{ CI 95 \% } & \multirow{2}{*}{ p value } \\
\cline { 3 - 4 } & & Lower & Upper & \\
\hline Age (per year increment) & 1.05 & 1.04 & 1.05 & $<0.001$ \\
BMI (per unit increment) & 0.92 & 0.90 & 0.93 & $<0.001$ \\
Male gender & 0.51 & 0.42 & 0.62 & $<0.001$ \\
NYHA status $>1$ & 1.07 & 0.96 & 1.18 & 0.22 \\
Height (per cm) & 0.99 & 0.98 & 1.00 & 0.01 \\
Creatinine (per 1 mg/dl) & 1.06 & 0.82 & 1.36 & 0.66 \\
Past MI & 1.19 & 1.05 & 1.36 & 0.01 \\
\hline
\end{tabular}

Abbreviations as in Table 1 multivariate model, no significant association with outcome was demonstrated. Gender and COPD were not associated with long-term outcomes. With regard to the bezafibrate treatment, we found that the distribution of subjects receiving fibrates was similar between the group with normal ALT values and the low-normal ALT group ( $22 \%$ vs. $23 \%$; $p=$ $0.67)$. Consistently addition of fibrate treatment as a covariant had no significant influence on the multivariate Cox model results. Therefore, it was excluded from our analysis.

\section{DISCUSSION}

The principle findings of this study are that, in patients with chronic CHD, low ALT levels are associated with older age,

Table 3 Independent Predictors of 22.8-year all-cause Mortality Outcome. **Model Further Adjusted for Serum Glucose, Alkaline Phosphatase and Triglyceride Concentrations. See Abbreviations in Table 1

\begin{tabular}{|c|c|c|c|c|}
\hline & \multirow{2}{*}{$\begin{array}{l}\text { Adjusted } \\
\text { hazard } \\
\text { ratio }\end{array}$} & \multicolumn{2}{|c|}{ CI $95 \%$} & \multirow[t]{2}{*}{$p$ value } \\
\hline & & Lower & Upper & \\
\hline ALT $<17$ IU/1 & 1.11 & 1.03 & 1.19 & 0.01 \\
\hline Age (per year increment) & 1.08 & 1.07 & 1.09 & $<0.001$ \\
\hline BMI (per unit increment) & 1.02 & 1.01 & 1.03 & $<0.001$ \\
\hline Male gender & 0.95 & 0.85 & 1.05 & 0.30 \\
\hline Current smoker & 1.71 & 1.54 & 1.91 & $<0.001$ \\
\hline Past smoker & 1.13 & 1.05 & 1.22 & 0.001 \\
\hline Hypertension & 1.09 & 1.02 & 1.17 & 0.01 \\
\hline Past MI & 1.43 & 1.32 & 1.54 & $<0.001$ \\
\hline COPD & 1.17 & 0.98 & 1.39 & 0.08 \\
\hline NYHA class $>1$ & 1.14 & 1.08 & 1.20 & $<0.001$ \\
\hline $\begin{array}{l}\text { Creatinine } \\
\text { (per mg/dl increment) }\end{array}$ & 1.61 & 1.38 & 1.87 & $<0.001$ \\
\hline
\end{tabular}




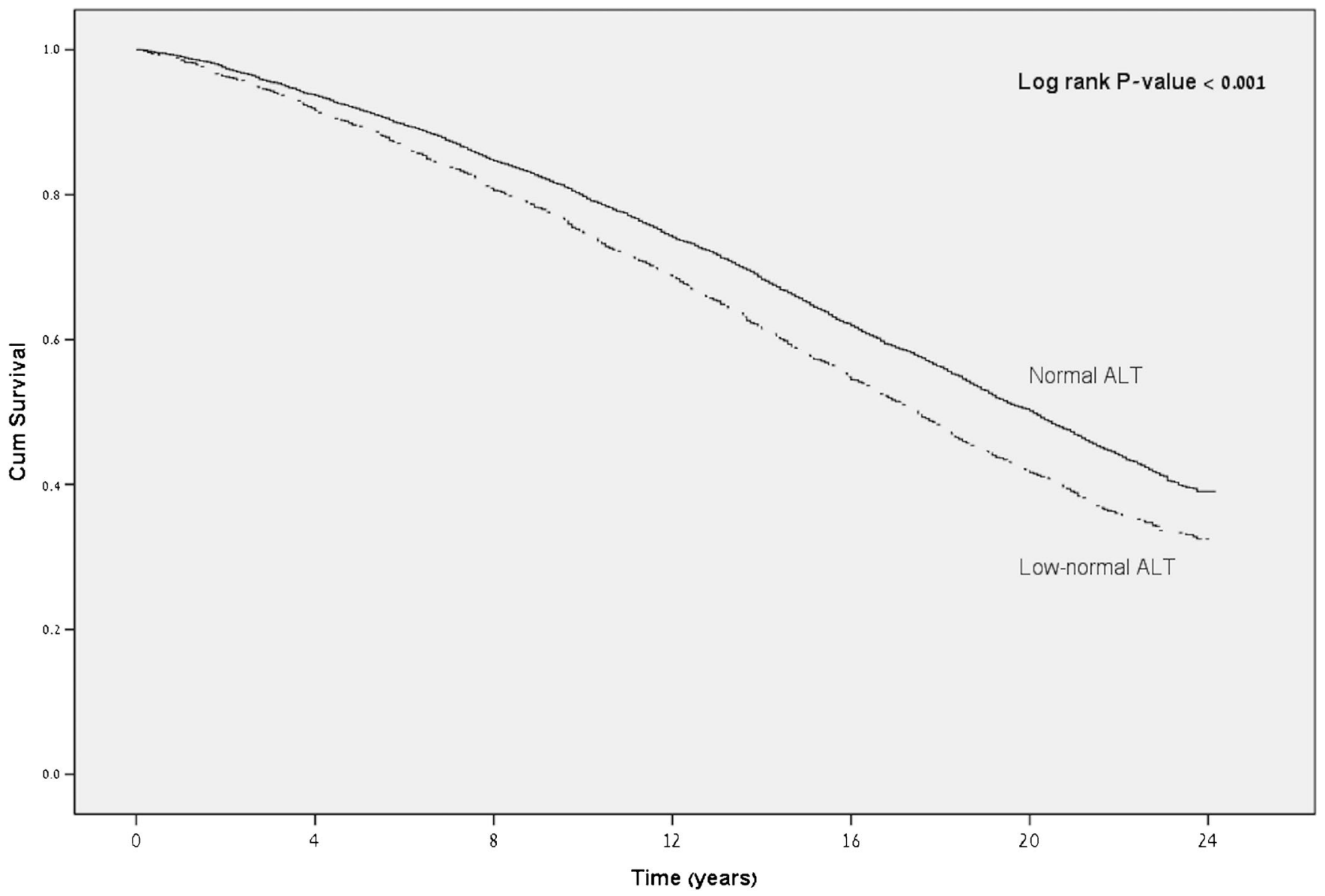

Figure 1 Cumulative survival probability at 22-year follow-up. $y$ axis: cumulative survival probability; $x$ axis: time (years).

female gender, lower BMI, lower height and a past MI. Patients with low ALT levels have increasingly greater risk of allcause long-term mortality with progressively lower ALT levels. Furthermore, the association of low ALT levels and mortality remained significantly independent following adjustment for multiple established predictors of mortality in this population. Thus, ALT levels may assist risk stratification in subjects with stable coronary disease.

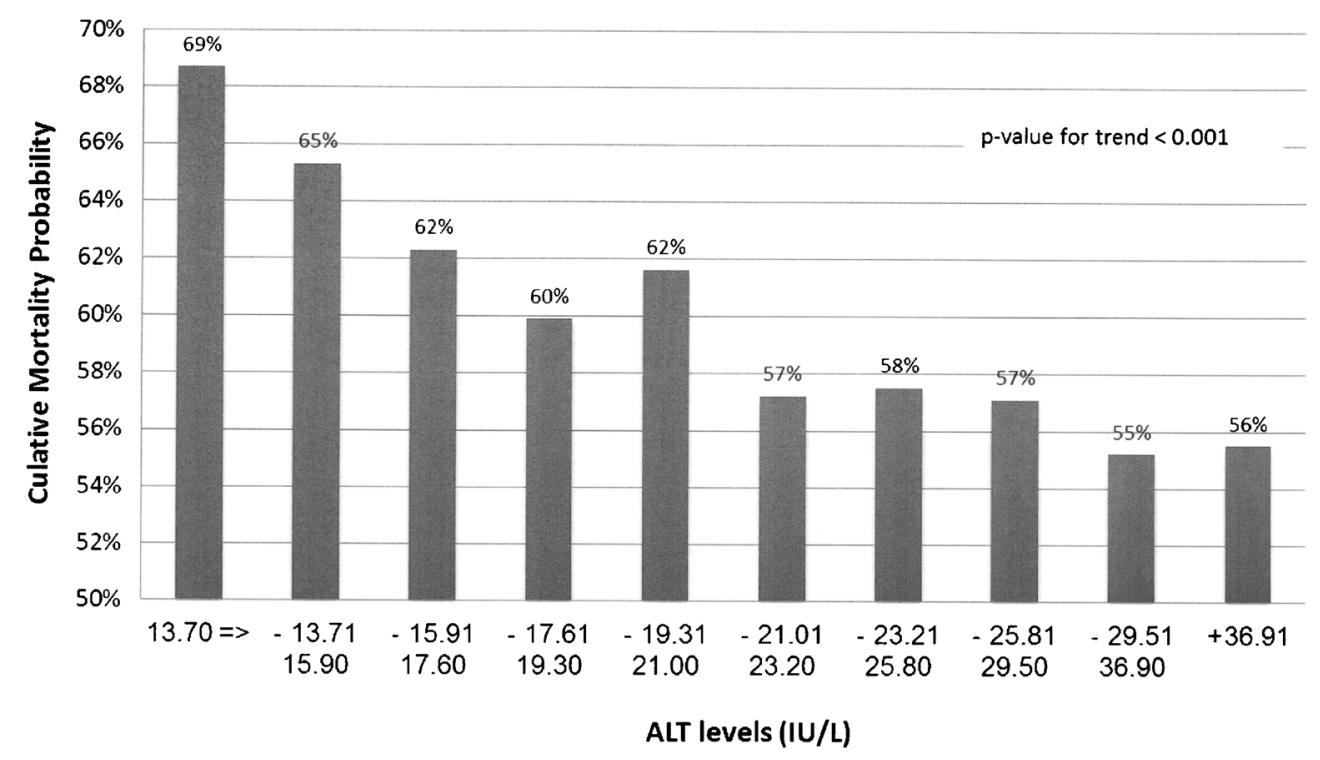

Figure 2 Mortality at 22-year follow-up by deciles of ALT levels within the normal range. $y$ axis: cumulative mortality probability; $x$ axis: ALT levels (IU/I). 
As the general population ages, diagnosing frailty becomes increasingly important - mainly among patient populations with common chronic diseases. One such patient population is the one included in the BIP registry: stable CHD patients. As these patients get older, increasingly advanced medications and invasive technologies are available to them (both as CHD and CHF patients). The ability to better diagnose their frailty and longevity is important for treatment planning in both the early and advanced stages of disease.

Low ALT blood levels have been shown to be associated with both frailty and shortened survival. In a recent study by Ramati et al., ${ }^{8}$ this was demonstrated in a population of healthy middle-aged adults. The fact that low ALT values and frailty lead to shortened survival is not necessarily the case for all adults, to say the least for adults suffering from chronic diseases, potentially shortening their life expectancy, at times leading to their death without a state of frailty on their path. Therefore, we found it important to address the relevant issue of low ALT as a biomarker for shortened survival in this patient (chronic IHD) population, which could have a shortened life span, without an obligatory state of frailty during their lifetime.

In the current study we have demonstrated a statistically significant association between low ALT levels of CHD patients and their long-term survival. The authors of the current study assume that the "missing link" between a low ALT value and survival is frailty. Therefore, we find it prudent to assume that low ALT values among stable CHD patients are associated with frailty and a shortened life expectancy.

It is assumed that a low ALT value serves as a biomarker for decreased muscular mass or sarcopenia in old age. The finding that low ALT values in middle-aged adults who are either healthy or suffering from chronic IHD are associated with a shortened life expectancy deserves special attention. The authors believe that a low ALT value might serve as an early predictor of frailty later in old age. It could be a part of an "early aging" syndrome, linking lower muscle mass in middleaged people with later development of frank sarcopenia in old age. This biologic hypothesis of course requirees further investigations.

Previous studies and reviews have addressed the possible direct and indirect association of ALT levels and CHD: in their study, Schooling et al. ${ }^{13}$ made multivariable proportional hazard regressions in 16,865 adults from the National Health and Nutrition Examination Survey (NHANES) III (1988-1994). They showed that ALT blood levels were positively associated with $\mathrm{CHD}$, which was considered to be associated with diabetes, but they were negatively associated with CHD, which was not associated with diabetes. They suggested that ALT levels, as a biomarker for liver function, may lead to an explanation regarding the paradox they described: the fact that stringent diabetes control does not reliably reduce cardiovascular events. The possible association with frailty was not addressed in their article. Schidhelm et al. ${ }^{14}$ demonstrated a positive correlation between high-normal ALT values and increased risk for coronary events in the general population, but no long-term correlation was addressed between low ALT values and the prognosis of CHD patients. None of the abovementioned publications addressed low ALT values as predictors of frailty and subsequent mortality in chronic CHD patients.

The current study has several limitations: it was conducted as a retrospective analysis of data; therefore, potential bias could not be ruled out and not all possible confounders could be accounted for. We have no information regarding the management and clinical events following the screening phase of the BIP registry. Also, we did not exclude special populations, potentially demonstrating increased or decreased ALT activity levels: Parkinson patients being treated with levodopa tend to have low-normal ALT levels since the enzyme responsible for dopamine degradation (dopamine carboxylase) utilizes the same cofactor as ALT does. ${ }^{15}$ On the other hand, different intake levels of vitamin B6 might interfere with the temporal results of ALT activity, not necessarily reflecting long-term ALT levels.

\section{CONCLUSIONS}

Low ALT blood levels may serve as an independent, longterm prognostic factor in the chronic CHD patient population. Serving as a quantitative parameter of frailty, ALT measurements may assist clinicians to build an overall patient assessment. Upon consideration of future surgery or interventional procedures, a low ALT value may serve as a marker of increased frailty and a poor long-term prognosis. Further prospective studies are warranted in order to better assess the contribution of ALT measurements in these settings.

Acknowledgments: No external or internal funding sources were used for this study.

Conflict of Interest: The authors declare that they do not have any conflict of interest.

Corresponding Author: G. Segal, MD; Department of Internal Medicine "T", Chaim Sheba Medical Center, Tel Hashomer, Ramat Gan, Israel (e-mail: gad.segal@sheba.health.gov.il).

\section{REFERENCES}

1. Le Couteur DG, et al. The association of alanine transaminase with aging, frailty, and mortality. J Gerontol A Biol Sci Med Sci. 2010;65(7):712-7.

2. Liu Z, et al. Alanine aminotransferase-old biomarker and new concept: a review. Int J Med Sci. 2014;11(9):925-35.

3. Ono K, Ono T, Matsumata T. The pathogenesis of decreased aspartate aminotransferase and alanine aminotransferase activity in the plasma of hemodialysis patients: the role of vitamin B6 deficiency. Clin Nephrol. 1995;43(6):405-8.

4. Yasuda $\mathbf{K}$, et al. Hypoaminotransferasemia in patients undergoing longterm hemodialysis: clinical and biochemical appraisal. Gastroenterology. 1995; 109(4): 1295-300.

5. Ensrud KE, et al. Comparison of 2 frailty indexes for prediction of falls, disability, fractures, and death in older women. Arch Intern Med. 2008;168(4):382-9. 
6. Fried LP, et al. Frailty in older adults: evidence for a phenotype. J Gerontol A Biol Sci Med Sci. 2001;56(3):M146-56.

7. Cesari M, et al. Sarcopenia and physical frailty: two sides of the same coin. Front Aging Neurosci. 2014;6:192.

8. Ramaty E, et al. Low ALT blood levels predict long-term all-cause mortality among adults. A historical prospective cohort study. Eur J Intern Med. 2014;25(10):919-21.

9. Goldbourt $\mathbf{U}$, et al. Lipids and lipoproteins in symptomatic coronary heart disease. Distribution, intercorrelations, and significance for risk classification in 6,700 men and 1,500 women. The Bezafibrate Infarction Prevention (BIP) Study Group, Israel. Circulation. 1992;86(3):839-48.

10. Goldbourt $\mathbf{U}$, et al. Rationale and design of a secondary prevention trial of increasing serum high-density lipoprotein cholesterol and reducing triglycerides in patients with clinically manifest atherosclerotic heart disease (the Bezafibrate Infarction Prevention Trial). Am J Cardiol. 1993;71(11):909-15.
11. Sanchis $\mathbf{J}$, et al. Frailty and other geriatric conditions for risk stratification of older patients with acute coronary syndrome. Am Heart J. 2014; 168(5):784-791. e2.

12. Rowe R, et al. Role of frailty assessment in patients undergoing cardiac interventions. Open Heart. 2014;1(1), e000033.

13. Schooling CM, Kelvin EA, Jones HE. Alanine transaminase has opposite associations with death from diabetes and ischemic heart disease in NHANES III. Ann Epidemiol. 2012;22(11):789-98.

14. Schindhelm RK, et al. Alanine aminotransferase predicts coronary heart disease events: a 10-year follow-up of the Hoorn Study. Atherosclerosis. 2007;191(2):391-6.

15. Bertoldi M. Mammalian Dopa decarboxylase: structure, catalytic activity and inhibition. Arch Biochem Biophys. 2014;546:1-7. 